\title{
Optimal Probabilistic Simulation of Quantum Channels from the Future to the Past
}

\author{
Dina Genkina, Giulio Chiribella, and Lucien Hardy \\ Perimeter Institute for Theoretical Physics, 31 Caroline Street North, Waterloo, Ontario N2L 2Y5, Canada.
}

(Dated: October 6, 2018)

\begin{abstract}
We introduce the study of quantum protocols that probabilistically simulate quantum channels from a sender in the future to a receiver in the past. The maximum probability of simulation is determined by causality and depends on the amount and type (classical or quantum) of information that the channel can transmit. We illustrate this dependence in several examples, including ideal classical and quantum channels, measure-and-prepare channels, partial trace channels, and universal cloning channels. For the simulation of partial trace channels, we consider generalized teleportation protocols that take $N$ input copies of a pure state in the future and produce $M \leq N$ output copies of the same state in the past. In this case, we show that the maximum probability of successful teleportation increases with the number of input copies, a feature that was impossible in classical physics. In the limit of asymptotically large $N$, the probability converges to the probability of simulation for an ideal classical channel. Similar results are found for universal cloning channels from $N$ copies to $M>N$ approximate copies, exploiting a time-reversal duality between universal cloning and partial trace.
\end{abstract}

\section{INTRODUCTION}

Quantum Theory is generally formulated relative to a given causal structure. This is the case both in Quantum Field Theory, where the spacetime metric is given from the beginning, and in the operational framework of Quantum Information, where protocols and computations consist in sequences of operations performed at different times. Relative to the given causal structure, Quantum Theory has to satisfy the causality principle [1, 2, stating that the probability of a measurement outcome at a given time be independent of the choice of operations performed at later times.

The causality principle forbids any form of signalling from the future to the past: A sender in the future cannot deterministically transfer the state of his system to a receiver in the past. However, it is easy to imagine situations where the state of a system is transferred from the future to the past with some probability, without leading to signalling. This is what happens, for example, if we eliminate the communication of classical data in the original quantum teleportation protocol [3]: The receiver in the past (say, Alice) can prepare a maximally entangled state and the sender in the future (say, Bob) can perform a Bell measurement on the state to be teleported together with half of the entangled state, as in Fig. 1. In this way, upon observing the right measurement outcome, Bob will know that the state has been teleported successfully to the past, and Alice will not need to perform any correction operation on the output. Of course, to know that teleportation has succeeded, Bob needs to wait until he sees the outcome of the Bell measurement. However, the important fact here is that, even before the measurement is performed, one half of the entangled state is already ready to be used by Alice in a quantum circuit: Alice can perform any desired computation on her system, and in the end, if teleportation is successful, Bob will know that Alice's computation has been performed on the input state he provided in the future. Conditionally to the

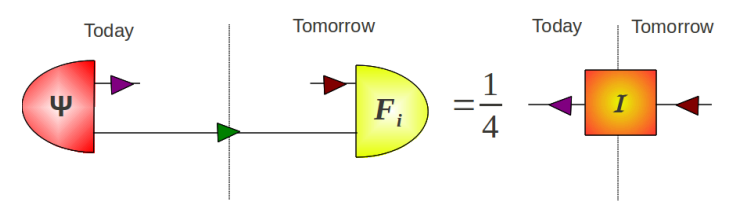

FIG. 1. (Color online) Probablilistic teleportation from the future to the past. Today Alice prepares a maximally entangled two-qubit state $\Psi$ of the ancilla and the output system, while tomorrow Bob performs a measurement on the ancilla and his input qubit. The measurement has four possible outcomes, for one of which (here denoted by $i_{0}$ ) successful teleportation will occur. With probability $p=\frac{1}{4}$ Alice obtains Bob's input state today. However, she will not know whether teleportation has been successful until Bob performs his measurement and communicates the outcome.

occurrence of the right outcome, everything behaves as though the input state has travelled from the future to the past through an ideal quantum channel. Note that this fact is not in contradiction with causality: The probability of teleportation is small enough that no signal can be sent from Bob to Alice. We will refer to the above use of probabilistic teleportation as a probabilistic simulation of the identity channel from the future to the past.

The idea of probabilistic teleportation as a simulated time-travel was originally proposed by Bennett and Schumacher in an unpublished work 4, and, more recently, by Svetlichny [5]. In different terms, this idea also appeared implicitly in the context of the graphical language of categorical quantum mechanics [, 9, where the reinterpretation of probabilistic teleportation as information flow from the future to the past follows from a stretching of wires in the basic teleportation diagram. Interestingly, similar ideas also appeared in the work of Horowitz and Maldacena [6] in the context of black hole evaporation, as an attempt to reconcile the unitarity of the black hole $S$ matrix with Hawking's semiclassical arguments (see also the discussion by Gottesmann and Preskill [7). Recently, 
the role of probabilistic teleportation in simulating closed timelike curves has been further explored by Lloyd et al in Refs. 10, 11]. In particular, Ref. [10] reports an experiment that uses probabilistic teleportation to simulate a quantum computation within a closed timelike curve. De Silva, Galvao and Kashefi [12] showed that some patterns in measurement-based quantum computation can be interpreted as deterministic simulations of closed timelike curves.

All the works mentioned so far focused on the use of teleportation for the probabilistic simulation of an ideal quantum channel from the future to the past. However, there are many interesting scenarios where one needs to consider the simulation of more general quantum channels. For example, suppose that we have $N$ identical copies of a the same state and that we want to teleport just one copy to the past. Does the probability of success increase with the number if input copies? And, if it does, what is the the asymptotic value of the success probability in the limit $N \rightarrow \infty$ ? To answer these questions we have to address the probabilistic simulation of channels that trace all systems but one.

In this paper we will address the general problem of the probabilistic simulation of a given channel from the future to the past, showing how the causality principle determines the maximum probability of success. To find the maximum probability we will optimize over all generalized teleportation schemes where a bipartite state is prepared and the input of the channel is jointly measured along with half of the bipartite state, so that, for a particular outcome, the desired channel is simulated, as in Fig. 2. The spirit of this work is similar to the spirit of

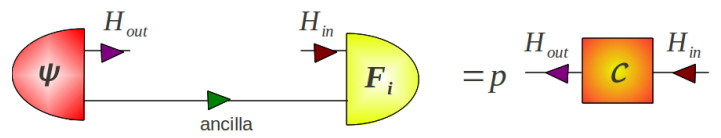

FIG. 2. (Color online) Probabilistic simulation of a given quantum channel from the future to the past via a generalized teleportation scheme. The output system and an ancilla are first prepared in a bipartite state $\Psi$. When the input becomes available, the input state and the ancilla are measured jointly. For a particular measurement outcome $i_{0}$ the simulation will be successful: The effective transformation from input to output will be proportional to the desired quantum channel, with proportionality constant $p$ equal to the probability of successful simulation.

the early works on the optimal cloning of non-orthogonal quantum states [13 17]: In that case, one knew from the no-cloning theorem [18, 19] that it is impossible to produce perfect copies of the input state and the goal was to find the optimal physical process that approximates the impossible cloning transformation. In our case, we know from causality that it is impossible to have a channel that deterministically transfers information from the future to the past and our goal is to find the optimal process that achieves the desired channel with maximum probability of success. In the same way in which the study of optimal cloning shed light on the process of copying information in the quantum world, we expect that the study of optimal simulations of channels to the past will shed light the interplay between causal structure and quantum information flow.

The main message of this paper is that the maximum probability of simulating a quantum channel from the future to the past is a decreasing function of the amount of information that the channel can transmit. This general feature will be illustrated in several examples. First, we will consider ideal classical channels (those that perfectly transmit the states of an orthonormal basis) and, second, ideal quantum channels (those that perfectly transmit all states of a given quantum system). In the first case we find the maximum probability

$$
p_{c l}=\frac{1}{d},
$$

where $d$ is the dimension of the Hilbert space, while in the second case we find

$$
p_{q}=\frac{1}{d^{2}},
$$

which is exactly the probability of the outcome that does not require correction operations in the original teleportation protocol [3]. We will then focus on the probabilistic simulation of measure-and-prepare channels, which are a class of channels that transmit only classical information. In this case, we will find that the maximum probability of success is at least equal to the probability $p_{c l}=1 / d$ for a classical channel with the same output Hilbert space. The exact value of the probability of success will be computed in two relevant cases of measureand-prepare channels: the channel associated to the optimal estimation of a pure state, and the universal NOT channel [20. Finally, we will analyze in detail the case of trace channels, sending $N$ copies of a given pure state to $M \leq N$ copies of the same state, and the case of universal cloning channels [15], sending $N$ copies of a pure state to $M>N$ optimal approximate copies. In both cases we find that the maximum probability of success is given by

$$
p_{q, N \rightarrow M}^{+}=\frac{d_{+}^{(|N-M|)}}{d_{+}^{(N)} d_{+}^{(M)}} \quad d_{+}^{(k)}:=\left(\begin{array}{c}
d+k-1 \\
k
\end{array}\right),
$$

where $d$ is the dimension of the one-particle Hilbert space and the superscript in $p_{q, N \rightarrow M}^{+}$reminds that we are restricting ourselves to the symmetric subspaces, in which the $N$ input copies and the $M$ output copies of the given pure state live. In the particular case of $M=1 \mathrm{Eq}$. (3) yields the value

$$
p_{q, N \rightarrow 1}^{+}=\frac{N}{d(d+N-1)},
$$

which increases with the number of input copies, starting from $p_{q, 1 \rightarrow 1}^{+}=1 / d^{2}$ and reaching the classical value 
$p_{c l}=1 / d$ in the asymptotic limit $N \rightarrow \infty$. This result has to be contrasted with the classical scenario, where having more input copies of a pure state cannot lead to any improvement: Since classical pure states can be perfectly cloned, there is no difference in having more input copies. The convergence of the probability of success to the classical value $p_{c l}=1 / d$ will be explained as a consequence of the convergence of the trace channel to a measure-and-prepare channel [21]. Concerning the probability $p_{q, N \rightarrow M}^{+}$in Eq. (3), it is also worth noting that it is symmetric in $N$ and $M$ : In other words, the probability of successful simulation for a trace channel from $N$ to $M \leq N$ copies is equal to the probability of successful simulation for a universal cloning channel from $M$ to $N \geq M$ copies. We will explain the symmetry of the probabilities as a consequence of a time-reversal duality between trace and universal cloning.

As we anticipated, the message emerging from our results is that the maximum probability for a given channel is a decreasing function of the amount of information that the channel can transmit: The smallest value $p_{q}=1 / d^{2}$ corresponds to the identity channel, while measure-andprepare channels have probability $p \geq 1 / d$, and the erasure channels $\mathcal{C}(\rho)=\operatorname{Tr}[\rho] \rho_{0}$ have probability $p=1$. We will also make the connection more quantitative, providing a set of statistical information bounds, stating that the probability of success in the simulation of a channel is upper bounded by the inverse of the amount of information that the channel can transmit. The amount of information will be quantified here as the maximum payoff that two parties can achieve in a communication game.

The structure of the paper is the following: In Section II we present the general method and, in particular, the causality bound, a necessary and sufficient condition for the existence of a probabilistic simulation with probability $p$. Then, we will analyze the probabilistic simulation of several channels: Sections III, IV, and V will focus on ideal classical and quantum channels, on measure-andprepare channels, and on partial trace channels from $N$ to $M \leq N$ copies, respectively. Section $\mathrm{V}$ will also discuss the case of universal cloning channels from $N$ to $M>N$ copies, exploiting the existence of a time-reversal duality between partial trace channels and cloning channels. Finally, in Section VI we will derive the statistical information bound and use it to explain the asymptotic behaviour of the maximum probability for partial trace and cloning channels. The conclusions of the paper are drawn in Section VII

\section{THEORETICAL FRAMEWORK}

To find the maximum probability to simulate a given channel we will use formalism of the Choi operators [22] and the framework of quantum combs [23, 24]. In this section we present the basic notions used in the paper and we derive the causality bound, a necessary and suffi- cient condition for the simulation of a given channel with probability $p$.

\section{A. Causality bound for quantum operations in a generalized teleportation protocol}

Any quantum circuit can be represented as a collection of preparations, measurements, transformations, and quantum wires along which states travel undisturbed. In this paper we only consider generalized teleportation circuits, which consist of one joint preparation of output and ancilla and one joint measurement on the input and ancilla, as in the circuit of Fig. 3 .

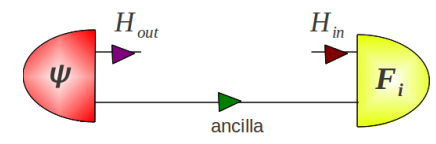

FIG. 3. (Color online) Generalized teleportation circuit consisting of a joint state preparation and of a joint measurement.

Here the preparation is represented by a joint quantum state $|\Psi\rangle \in \mathcal{H}_{A} \otimes \mathcal{H}_{\text {out }}$ of the ancilla and the output system, and the quantum measurement is represented by a positive operator valued measure (POVM) $\left\{F_{i}\right\}_{i \in X}$, namely a collection of positive operators $F_{i} \geq 0$ acting on the Hilbert space $\mathcal{H}_{i n} \otimes \mathcal{H}_{A}$ with the normalization property

$$
\sum_{i \in X} F_{i}=I_{i n} \otimes I_{A}
$$

$I_{\text {in }}$ and $I_{A}$ representing the identity operators on the input Hilbert space $\mathcal{H}_{i n}$ and on the ancilla Hilbert space $\mathcal{H}_{A}$, respectively.

Each possible outcome $i \in X$ corresponds to a different linear map $\mathcal{E}_{i}$, mapping density matrices on the input Hilbert space $\mathcal{H}_{\text {in }}$ to density matrices on the output Hilbert space $\mathcal{H}_{\text {out }}$, and given by

$$
\mathcal{E}_{i}(\rho):=\operatorname{Tr}_{\text {in }, A}\left[\left(F_{i} \otimes I_{\text {out }}\right)(\rho \otimes|\Psi\rangle\langle\Psi|)\right]
$$

$\operatorname{Tr}_{i n, A}$ denoting the partial trace over the Hilbert spaces $\mathcal{H}_{\text {in }}$ and $\mathcal{H}_{A}$. By construction, each linear map $\mathcal{E}_{i}$ is completely positive and trace non-increasing, namely it is a quantum operation [25. Note that, however, in this case the output of the quantum operation precedes the input in time, differing to the standard case originally considered by Kraus 25.

Consider now the sum over all possible maps $\left\{\mathcal{E}_{i}\right\}_{i \in X}$ corresponding to different measurement outcomes. Summing over all possible measurement outcomes is equivalent to ignoring the measurement outcome, thus obtaining the completely positive trace-preserving map (also 
known as quantum channel) $\mathcal{E}_{X}$ given by

$$
\begin{aligned}
\mathcal{E}_{X}(\rho) & =\sum_{i \in X} \mathcal{E}_{i}(\rho) \\
& =\sum_{i \in X} \operatorname{Tr}_{\text {in }, A}\left[\left(F_{i} \otimes I_{\text {out }}\right)(\rho \otimes|\Psi\rangle\langle\Psi|)\right] \\
& =\sum_{i \in X} \operatorname{Tr}_{\text {in }, A}[\rho \otimes|\Psi\rangle\langle\Psi|] \\
& =\rho_{0} \operatorname{Tr}_{\text {in }}[\rho] \quad \rho_{0}:=\operatorname{Tr}_{A}[|\Psi\rangle\langle\Psi|],
\end{aligned}
$$

the third equality coming from the normalization of Eq. (5). Note that the trace-preserving map $\mathcal{E}_{X}$ is an erasure channel: It always produces the same output state $\rho_{0}$ independently of the input. The fact that $\mathcal{E}_{X}$ is an erasure channel is a manifestation of the causality principle stating that no signal can be sent from the future back to the past. In the following we will use the notation $\mathcal{E}_{X}=\rho_{0} \operatorname{Tr}_{i n}$ to mean that $\mathcal{E}_{X}$ is the map defined by $\mathcal{E}_{X}(\rho)=\rho_{0} \operatorname{Tr}_{i n}[\rho]$, for every quantum state $\rho$ on $\mathcal{H}_{i n}$.

Since the linear maps $\left\{\mathcal{E}_{i}\right\}_{i \in X}$ are all completely positive, we must have

$$
\mathcal{E}_{i} \leq \rho_{0} \operatorname{Tr}_{i n} \quad \forall i \in X
$$

where the inequality $\mathcal{A} \leq \mathcal{B}$ for two linear maps $\mathcal{A}$ and $\mathcal{B}$ means that the linear map $\mathcal{B}-\mathcal{A}$ is completely positive. We will refer to Eq. (6) as the causality bound for a quantum operation $\mathcal{E}_{i}$ in a generalized teleportation scheme.

We can now focus on the a probabilistic simulation of a given quantum channel $\mathcal{C}$ from the future to the past. In this case we want that for one particular measurement outcome $i_{0} \in X$ the transformation $\mathcal{E}_{i_{0}}$ is proportional to $\mathcal{C}$, namely $\mathcal{E}_{i_{0}}=p \mathcal{C}$. In this case, the proportionality constant $p$ represents the probability that the simulation succeeds. The maximum value of the success probability $p$ must be compatible with the causality bound

$$
p \mathcal{C} \leq \rho_{0} \operatorname{Tr}_{\mathrm{in}}
$$

We will refer to the above bound as the causality bound for the simulation of channel $\mathcal{C}$. An obvious consequence of the causality bound is the following:

Proposition 1 A channel $\mathcal{C}$ can be simulated with probability $p=1$ in a generalized probabilistic teleportation protocol if and only if $\mathcal{C}$ is an erasure channel, namely $\mathcal{C}=\rho_{0} \operatorname{Tr}_{i n}$.

Proof. Suppose that we have $p=1$ in the causality bound of Eq. (7) and define the quantum operation $\mathcal{D}:=$ $\rho_{0} \operatorname{Tr}_{i n}-\mathcal{C}$, so that we have $\mathcal{C}+\mathcal{D}=\rho_{0} \operatorname{Tr}_{i n}$. Applying both sides of the equality to a generic state $\rho$ and taking the trace, we obtain $\operatorname{Tr}[\mathcal{C}(\rho)]+\operatorname{Tr}[\mathcal{D}(\rho)]=\operatorname{Tr}[\rho]$. Since $\mathcal{C}$ is a quantum channel, by definition $\operatorname{Tr}[\mathcal{C}(\rho)]=\operatorname{Tr}[\rho]$. That is, $\operatorname{Tr}[\mathcal{D}(\rho)]=0$ for every $\rho$. Hence, we conclude that $\mathcal{D}=0$, and, therefore $\mathcal{C}=\rho_{0} \operatorname{Tr}_{i n}$.

In the following section we will show that the validity of the causality bound is not only necessary, but also sufficient for the existence of a probabilistic simulation with probability $p$.

\section{B. Causality bound on Choi operators}

A very convenient tool to study quantum operations and channels is the Choi correspondence, which associates linear operators on he Hilbert space $\mathcal{H}_{\text {out }} \otimes \mathcal{H}_{\text {in }}$ to linear maps sending operators on $\mathcal{H}_{\text {in }}$ to operators on $\mathcal{H}_{\text {out }}$. The Choi operator $E$ corresponding to a linear $\operatorname{map} \mathcal{E}$ is given by

$$
E=\left(\mathcal{E} \otimes \mathcal{I}_{\text {in }}\right)(|I\rangle\rangle\langle\langle I|)
$$

where $|I\rangle\rangle$ indicates the unnormalized maximally entangled state $\sum_{i}|i\rangle \otimes|i\rangle,\left\{|i\rangle \mid i=1, \ldots, d_{i n}\right\}$ being an orthonormal basis for $\mathcal{H}_{i n}$.

We will often use the "double ket" notation of Ref. 26] for bipartite states on a tensor product Hilbert space $\mathcal{H} \otimes \mathcal{K}:$ This notation is based on the one-to-one correspondence between operators $C$ from $\mathcal{K}$ to $\mathcal{H}$ and states $|C\rangle\rangle$ in $\mathcal{H} \otimes \mathcal{K}$, given by

$$
|C\rangle\rangle:=\sum_{i=1}^{d_{\mathcal{H}}} \sum_{j=1}^{d_{\mathcal{K}}}\left\langle\varphi_{i}|C| \psi_{j}\right\rangle\left|\varphi_{i}\right\rangle \otimes\left|\psi_{j}\right\rangle,
$$

where $\left\{\left|\varphi_{i}\right\rangle\right\}_{i=1}^{d_{\mathcal{H}}}$ is an orthonormal basis for $\mathcal{H}$ and $\left\{\left|\psi_{j}\right\rangle\right\}_{j=1}^{d_{\mathcal{K}}}$ is an orthonormal basis for $\mathcal{K}$. The "double ket" notation has the useful properties

$$
\langle\langle C \mid D\rangle\rangle=\operatorname{Tr}\left[C^{\dagger} D\right]
$$

for every pair of operators $C, D$ from $\mathcal{K}$ to $\mathcal{H}$, and

$$
\left.(A \otimes B)|C\rangle\rangle=\left|A C B^{T}\right\rangle\right\rangle,
$$

for every operator $A$ on $\mathcal{H}, B$ on $\mathcal{K}$, and $C$ from $\mathcal{K}$ to $\mathcal{H}$. Here $B^{T}:=\sum_{i, j}\left\langle\psi_{i}|B| \psi_{j}\right\rangle\left|\psi_{j}\right\rangle\left\langle\psi_{i}\right|$ denotes the transpose of $B$ in the given basis $\left\{\left|\psi_{j}\right\rangle\right\}_{j=1}^{d_{\mathcal{K}}}$.

Since the correspondence between linear maps and Choi operators is one-to-one every property of a linear map corresponds to a property of the associated Choi operator. In particular, we have Choi's theorem, stating that a linear map $\mathcal{E}$ is completely positive if and only if its Choi operator $E$ is positive semi-definite [22]. Moreoever, a linear map is trace preserving if and only if its Choi operator obeys $\operatorname{Tr}_{\text {out }}[E]=I_{\text {in }}$.

Let us now re-cast the causality bound of Eq. (7) in terms of Choi operators. The Choi operator of the erasure channel $\mathcal{E}_{X}=\rho_{0} \operatorname{Tr}_{i n}$ is given by

$$
\begin{aligned}
E_{X} & =\left(\mathcal{E}_{X} \otimes \mathcal{I}_{i n}\right)(|I\rangle\rangle\langle\langle I|) \\
& =\rho_{0} \otimes I_{i n} .
\end{aligned}
$$

Denoting by $C$ the Choi operator of the quantum channel $\mathcal{C}$, the causality bound of Eq. (7) becomes

$$
p C \leq \rho_{\text {out }} \otimes I_{\text {in }}
$$

This operator form of the causality bound is much simpler to handle than the version with linear maps. In the 
following we will use Eq. 11 to find the maximum probability $p$ for the simulation of a channel $\mathcal{C}$ to the past.

We now use the method of quantum combs [23, 24 to show that any value of $p$ compatible with the causality bound of Eq. (11) can be achieved in a suitable generalized teleportation protocol. The key result that we use is Theorem 4 of Ref. 24, whose statement, specialized to the case considered in this paper, can be rephrased as

Lemma 1 Let $\left\{E_{i}\right\}_{i \in X}$ be a collection of positive operators on $\mathcal{H}_{\text {out }} \otimes \mathcal{H}_{\text {in }}$ satisfying the property $\sum_{i \in X} E_{i}=$ $\rho_{0} \otimes I_{\text {in }}$ for some state $\rho_{0}$ on $\mathcal{H}_{\text {out }}$. Then, there exists an ancillary system $A$, a pure state $\Psi \in \mathcal{H}_{A} \otimes \mathcal{H}_{\text {out }}$, and a POVM $\left\{F_{i}\right\}_{i \in X}$ on $\mathcal{H}_{i n} \otimes \mathcal{H}_{A}$ such that, for every $i \in X$, $E_{i}$ is the Choi operator of the quantum operation $\mathcal{E}_{i}$ given by

$$
\mathcal{E}_{i}(\rho):=\operatorname{Tr}_{\text {in }, A}\left[(\rho \otimes|\Psi\rangle\langle\Psi|)\left(F_{i} \otimes I_{\text {out }}\right)\right]
$$

for every state $\rho$ on $\mathcal{H}_{\text {in }}$.

A simple consequence of lemma 1 is that every value of $p$ compatible with the causality bound is attainable in some generalized teleportation protocol:

Theorem 1 (Achievability of the causality bound) Let $\mathcal{C} \in \operatorname{Lin}\left(\mathcal{H}_{\text {out }} \otimes \mathcal{H}_{\text {in }}\right)$ be the Choi operator of the channel $\mathcal{C}$. If there exists a state $\rho_{0}$ on $\mathcal{H}_{\text {out }}$ such that $p C \leq \rho_{0} \otimes I_{i n}$, then there exists a generalized teleportation scheme such that simulates $\mathcal{C}$ with probability $p$.

Proof. To prove the thesis it is enough to define $E_{0}:=$ $p C$ and $E_{1}:=\rho_{0} \otimes I_{i n}-p C$ and to apply lemma 1 to the collection of operators $\left\{E_{0}, E_{1}\right\}$. The simulation of channel $\mathcal{C}$ will succeed when the measurement outcome is 0 .

Thanks to theorem 1 we can derive the maximum probability of successful simulation from the causality bound of Eq. 111): We will know automatically that there exists a generalized teleportation protocol succeeding with that maximum probability.

\section{IDEAL CHANNELS}

In this Section we will illustrate the general method of the causality bound in two simple examples. The first example is about the probabilistic simulation of an ideal classical channel, which preserves the elements of an orthonormal basis, but destroys all off-diagonal elements of the density matrix. In this case, we will show that the probability of success is given by

$$
p_{c l}=\frac{1}{d}
$$

where $d$ is the dimension of the Hilbert space. The second example is about the probabilistic simulation of an ideal quantum channel, represented by the identity map. In this case, using the causality bound we will show that the probability of success is given by

$$
p_{q}=\frac{1}{d^{2}}
$$

$d$ being again dimension of the Hilbert space. This also proves that the original teleportation protocol [3] is optimal for the probabilistic simulation of an ideal channel from the future to the past.

\section{A. Optimal probabilistic simulation of an ideal classical channel from the future to the past}

An ideal classical channel can be modelled in Quantum Theory as a von Neumann measurement on an orthonormal basis, represented by the channel $\mathcal{C}_{c l}(\rho)=$ $\sum_{i=1}^{d}|i\rangle\langle i|\langle i|\rho| i\rangle$, where $\rho$ is the state of a $d$-dimensional quantum system. By the definition of Choi operator in Eq. (8), the Choi operator of $\mathcal{C}_{c l}$ is given by

$$
C_{c l}=\sum_{i=1}^{d}|i\rangle\langle i|\otimes| i\rangle\langle i| .
$$

Inserting the above expression in the causality bound of Eq. (11), we obtain

$$
p\left(\sum_{i=1}^{d}|i\rangle\langle i|\otimes| i\rangle\langle i|\right) \leq \rho_{\text {out }} \otimes I_{\text {in }} .
$$

Then, taking on both sides the expectation value on the state $|i\rangle \otimes|i\rangle$, we get $p \leq\left\langle i\left|\rho_{\text {out }}\right| i\right\rangle$, for every $i=1, \ldots, d$, which also implies $p \leq \min _{1 \leq i \leq d}\left\langle i\left|\rho_{\text {out }}\right| i\right\rangle \leq \frac{1}{d}$. Choosing $\rho_{\text {out }}=\frac{I_{\text {out }}}{d}$ we then achieve the maximum value

$$
p_{c l}=\frac{1}{d} .
$$

A generalized teleportation protocol that simulates the ideal classical channel with maximum probability $p_{c l}=\frac{1}{d}$ can be easily obtained by preparing the classically correlated state

$$
\sigma_{c l}=\frac{\sum_{i}|i\rangle\langle i|\otimes| i\rangle\langle i|}{d}
$$

and by measuring the two-outcome POVM $\left\{P_{0}, P_{1}\right\}$ given by $P_{0}:=\sum_{i}|i\rangle\langle i|\otimes| i\rangle\langle i|$ and $P_{1}:=I-P_{0}$.

\section{B. Optimal probabilistic simulation of an ideal quantum channel from the future to the past}

Another instructive application of the general method is the proof that the value of the probability of successful teleportation $p_{q}=\frac{1}{d^{2}}$ in the original teleportation protocol [3] is the optimal value determined by causality. 
In the case of teleportation, the quantum channel that we want to simulate is just the identity: $\mathcal{C}_{q}=\mathcal{I}$. By definition of Choi operator Eq. (8), the Choi operator of the identity channel is

$$
\left.C_{q}=|I\rangle\right\rangle\langle\langle I| .
$$

Plugging this into the causality bound of Eq. (11), we then obtain

$$
p|I\rangle\rangle\left\langle\langle I| \leq \rho_{\text {out }} \otimes I_{\text {in }} .\right.
$$

To find the maximum value of the simulation probability $p$ we must ensure that the difference between the right hand side and the left hand side is a positive operator. In general, this is not a trivial issue because the operators on the two sides are not necessarily diagonalizable in the same basis.

To resolve this problem, we notice a symmetry of the left hand side. Defining the complex conjugate of an operator as $A^{*}:=\sum_{i, j}\langle i|A| j\rangle^{*}|i\rangle\langle j|$ and using Eq. 10 we have

$$
\left.\left.\left(U \otimes U^{*}\right)|I\rangle\right\rangle=|I\rangle\right\rangle \quad \forall U \in S U(d) .
$$

and, therefore, $\left(U \otimes U^{*}\right) C_{q}\left(U \otimes U^{*}\right)^{\dagger}=C_{q} \quad \forall U \in S U(d)$. Applying the same transformation on both sides of the inequality (14), we obtain

$$
p|I\rangle\left\langle\langle I| \leq U \rho_{\text {out }} U^{\dagger} \otimes I_{\text {in }} \quad \forall U \in S U(d) .\right.
$$

Since the inequality must hold for every unitary $U \in$ $S U(d)$, it also holds for the integral over the normalized Haar measure $d U$,

$$
\begin{aligned}
p|I\rangle\rangle\langle\langle I| & \leq \int d U\left(U \rho U^{\dagger} \otimes I_{\text {in }}\right) \\
& =\frac{I_{\text {out }} \otimes I_{\text {in }}}{d},
\end{aligned}
$$

having used the Schur's lemma for the last equality. Now, both sides of the inequality are diagonalizable in the same basis. Since the operator on the left hand side has eigenvalue $p d$ and the operator on the right hand side has eigenvalue of $\frac{1}{d}$, the inequality becomes $p d \leq \frac{1}{d}$. Hence, the maximum probability of teleportation compatible with the causality bound is

$$
p_{q}=\frac{1}{d^{2}}
$$

Thus, we have shown from causality that the original teleportation protocol is the optimal probabilistic simulation of an identity channel from the future to the past.

\section{Lower bound for general channels}

The probability of success for ideal channels provides a lower bound for the probability of success for arbitrary channels: Indeed, if we have protocols that simulate ideal channels from the future to the past, then we can use these protocols to simulate a desired channel $\mathcal{C}$ in two ways: $i$ ) we can apply $\mathcal{C}$ to the input in the future and then send the output to the past through the ideal channel, or ii) we can send the input to the past through the ideal channel and apply the desired channel $\mathcal{C}$ to the input in the past. This argument leads to the following:

Proposition 2 Let $\mathcal{C}$ be a quantum channel sending states on the Hilbert space $\mathcal{H}_{\text {in }}$ to states on the Hilbert space $\mathcal{H}_{\text {out }}$. Then, the maximum probability $p_{\mathcal{C}}$ to simulate the action of $\mathcal{C}$ from the future to the past satisfies the bound

$$
p_{\mathcal{C}} \geq \max \left\{\frac{1}{d_{\text {in }}^{2}}, \frac{1}{d_{\text {out }}^{2}}\right\} .
$$

For classical channels, of the form $\mathcal{C}(\rho)=$ $\sum_{i, j} p(j \mid i) \quad\left|\psi_{j}\right\rangle\left\langle\psi_{j}\right|\left\langle\varphi_{i}|\rho| \varphi_{i}\right\rangle$ where $\left\{\left|\varphi_{i}\right\rangle\right\}_{i=1}^{d_{i n}}$ and $\left\{\left|\psi_{j}\right\rangle\right\}_{j=1}^{d_{\text {out }}}$ are orthonormal bases for $\mathcal{H}_{\text {in }}$ and $\mathcal{H}_{\text {out }}$, respectively, the bound becomes

$$
p_{\mathcal{C}} \geq \max \left\{\frac{1}{d_{\text {in }}}, \frac{1}{d_{\text {out }}}\right\} .
$$

\section{MEASURE-AND-PREPARE CHANNELS}

In this section we focus on measure-and-prepare channels, namely channels $\mathcal{C}_{m \& p}$ of the form

$$
\mathcal{C}_{m \& p}(\rho)=\sum_{i \in X} \rho_{i} \operatorname{Tr}\left[P_{i} \rho\right],
$$

where $\left\{P_{i}\right\}_{i \in X}$ is a POVM on the input Hilbert space $\mathcal{H}_{\text {in }}$, that is,

$$
P_{i} \geq 0 \quad \forall i \in X, \quad \sum_{i \in X} P_{i}=I_{\text {in }}
$$

and $\left\{\rho_{i}\right\}_{i \in X}$ is a collection of normalized density matrices on the output Hilbert space $\mathcal{H}_{\text {out }}$, that is,

$$
\rho_{i} \geq 0, \quad \operatorname{Tr}\left[\rho_{i}\right]=1 \quad \forall i \in X .
$$

Measure-and-prepare channels are interesting as an example of channels that transmit only classical information: Indeed, it is well known that the quantum capacity of a measure-and-prepare channel is always zero (this follows, e.g. from the general upper bound presented by Holevo and Werner in Ref. [27]).

In this section we will first show that the maximum probability to simulate a given measure-and-prepare channel from the future to the past satisfies the general lower bound

$$
p_{m \& p} \geq \frac{1}{d_{\text {out }}},
$$

where $d_{\text {out }}$ is the dimension of the output Hilbert space. The bound is achieved for $\mathcal{H}_{\text {in }}=\mathcal{H}_{\text {out }}$ by von Neumann 
measurements on an orthonormal basis, and in that case coincides with the bound for the simulation of an ideal classical channel (cf. subsection III A). Eq. (18) is quite intuitive: If a channel can only transmit classical information, then the probability to simulate it is at least equal to the probability to simulate an ideal classical channel with the same output.

Then we will compute the exact value of the probability of success for two particular examples of measureand-prepare channels: The first example will be the channel associated to the optimal estimation of a pure state, namely the linear map $\mathcal{C}_{\text {est }}$ sending states on $\mathcal{H}$ to states on $\mathcal{H}$ given by

$$
\begin{aligned}
& \mathcal{C}_{\text {est }}(\rho)=\int d U \rho_{U} \operatorname{Tr}\left[P_{U} \rho\right] \quad \rho_{U}:=U|0\rangle\langle 0| U^{\dagger} \\
& P_{U}:=d U|0\rangle\langle 0| U^{\dagger},
\end{aligned}
$$

where $d$ is the dimension of $\mathcal{H}$ and $|0\rangle \in \mathcal{H}$ is a fixed state. In this case the general bound is tight: the probability of success is given by

$$
p_{\text {est }}=\frac{1}{d}
$$

The second example will consist in the universal NOT channel [20], namely the linear map $\mathcal{C}_{U-N O T}$ sending states on $\mathcal{H}$ to states on $\mathcal{H}$ given by

$$
\begin{aligned}
& \mathcal{C}_{U-N O T}(\rho)=\int d U \rho_{U}^{\perp} \operatorname{Tr}\left[P_{U} \rho\right] \rho_{U}^{\perp}:=\frac{U(I-|0\rangle\langle 0|) U^{\dagger}}{d-1} \\
& P_{U}:=d U|0\rangle\langle 0| U^{\dagger}
\end{aligned}
$$

In this case we will show that the probability of success is given by

$$
p_{U-N O T}=1-\frac{1}{d^{2}},
$$

well above the lower bound $p_{m \& p} \geq \frac{1}{d}$. The intuition that the probability of success depends on the amount of information that a channel can transmit suggests that the universal NOT channel can transmit less classical information than the state estimation channel.

\section{A. General lower bound on the probability of success}

The Choi operator of the generic measure-and-prepare channel $\mathcal{C}_{m \& p}$ in Eq. (17) is given by

$$
C_{m \& p}=\sum_{i \in X}\left(\rho_{i} \otimes P_{i}^{T}\right) .
$$

Let us consider the causality bound $p C_{m \& p} \leq \rho_{0} \otimes I_{i n}$, where $\rho_{0}$ is a state on $\mathcal{H}_{\text {out }}$ to be optimized. A (possibly suboptimal) choice for the state $\rho_{0}$ is $\rho_{0}=\frac{I_{\text {out }}}{d_{\text {out }}}$, for which the bound becomes

$$
p C_{m \& p} \leq \frac{I_{\text {out }} \otimes I_{\text {in }}}{d_{\text {out }}} .
$$

The bound will be satisfied as long as we choose $p=$ $\frac{1}{d_{\text {out }} \gamma_{\max }}$, where $\gamma_{\max }$ is the largest eigenvalue of the Choi operator $C_{m \& p}$.

We now show that $\gamma_{\max }$ cannot be larger than 1 . To this purpose, we consider an arbitrary normalized state $|\Psi\rangle\rangle \in \mathcal{H}_{\text {out }} \otimes \mathcal{H}_{\text {in }}$ and show that $\left\langle\left\langle\Psi\left|C_{m \& p}\right| \Psi\right\rangle\right\rangle \leq 1$. Using Eq. (9), the normalization of the state $|\Psi\rangle\rangle$ reads

$$
\langle\langle\Psi \mid \Psi\rangle\rangle=\operatorname{Tr}\left[\Psi^{\dagger} \Psi\right]=1
$$

We then obtain

$$
\begin{aligned}
\left\langle\left\langle\Psi\left|C_{m \& p}\right| \Psi\right\rangle\right\rangle & =\sum_{i \in X}\left\langle\left\langle\Psi \mid \rho_{i} \Psi P_{i}\right\rangle\right\rangle \\
& =\sum_{i \in X} \operatorname{Tr}\left[\Psi^{\dagger} \rho_{i} \Psi P_{i}\right] . \\
& =\sum_{i}^{\prime} \operatorname{Tr}\left[\Psi P_{i} \Psi^{\dagger}\right] \operatorname{Tr}\left[\rho_{i} \sigma_{i}\right] \quad \sigma_{i}:=\frac{\Psi P_{i} \Psi^{\dagger}}{\operatorname{Tr}\left[\Psi P_{i} \Psi^{\dagger}\right]} \\
& \leq \sum_{i \in X} \operatorname{Tr}\left[\Psi P_{i} \Psi^{\dagger}\right] \\
& =\operatorname{Tr}\left[\Psi^{\dagger} \Psi\right] \\
& =1 .
\end{aligned}
$$

Here we used Eq. 10 for the first equality and Eq. (9) for the second. The sum in the third equation is restricted to the values of $i$ such that $\operatorname{Tr}\left[\Psi P_{i} \Psi^{\dagger}\right] \neq 0$. The inequality is due to the fact that the overlap between the density matrices $\rho_{i}$ and $\sigma_{i}$ is bounded by 1 , the fourth equality is due to the normalization condition $\sum_{i \in X} P_{i}=$ $I_{i n}$, and the last equality is just Eq. 21.

Since the bound $\left\langle\left\langle\Psi\left|C_{m \& p}\right| \Psi\right\rangle\right\rangle \leq 1$ holds for arbitrary $|\Psi\rangle\rangle$, we conclude that the maximum eigenvalue of $C_{m \& p}$ satisfies $\gamma_{\max } \leq 1$. This implies that the value $p=1 / d_{\text {out }}$ is compatible with the causality bound, and therefore, it is achievable with a suitable generalized teleportation protocol. Hence, for the maximum probability $p_{m \& p}$ we have the lower bound $p_{m \& p} \geq \frac{1}{d_{\text {out }}}$.

\section{B. Optimal pure state estimation}

The Choi operator for the pure state estimation channel is

$$
\begin{aligned}
C_{e s t} & =d \int d U\left(U \otimes U^{*}\right)(|0\rangle\langle 0|\otimes| 0\rangle\langle 0|)\left(U^{\dagger} \otimes U^{T}\right) \\
& \left.=\frac{1}{d}|I\rangle\right\rangle\left\langle\langle I|+\frac{1}{d+1}\left(I \otimes I-\frac{|I\rangle\rangle\langle\langle I|}{d}\right) .\right.
\end{aligned}
$$

Clearly, $C_{\text {est }}$ enjoys the symmetry $\left(U \otimes U^{*}\right) C_{\text {est }}\left(U^{\dagger} \otimes\right.$ $\left.U^{T}\right)=C_{\text {est }}$ for every $U \in S U(d)$, which implies that the 
causality bound has the form

$$
p C_{e s t} \leq \frac{I \otimes I}{d}
$$

(the proof is the same as the proof for the ideal quantum channel in subsection III B.

Since the maximum eigenvalue of $C_{e s t}$ is $\gamma_{\max }=1$, we have that the maximum probability is $p_{\text {est }}=\frac{1}{d}$.

\section{Universal NOT channel}

Using Eqs. (19) and (20), the universal NOT channel can be written as

$$
\mathcal{C}_{U-N O T}=\frac{1}{d-1}\left(I \operatorname{Tr}-\mathcal{C}_{e s t}\right) .
$$

Hence, its Choi operator is given by

$$
\begin{aligned}
C_{U-N O T} & =\frac{1}{d-1}\left(I \otimes I-C_{e s t}\right) \\
& =\frac{d}{d^{2}-1}\left(I \otimes I-\frac{|I\rangle\rangle\langle\langle I|}{d}\right) .
\end{aligned}
$$

Again, we observe that $C_{U-N O T}$ enjoys the symmetry $\left(U \otimes U^{*}\right) C_{U-N O T}\left(U^{\dagger} \otimes U^{T}\right)=C_{U-N O T}$ for every $U \in$ $S U(d)$, which implies that the causality bound has the form

$$
p C_{U-N O T} \leq \frac{I \otimes I}{d} .
$$

Now, the maximum eigenvalue of $C_{U-N O T}$ is $\gamma_{\max }=$ $\frac{d}{d^{2}-1}$. Hence, the maximum probability is given by $p_{U-N O T}=\frac{1}{d \gamma_{\max }}=1-\frac{1}{d^{2}}$.

\section{OPTIMAL PROBABILISTIC TELEPORTATION WITH MULTIPLE INPUT AND OUTPUT COPIES}

In this section we consider probabilistic protocols that take $N$ input copies of a pure state in the future and produce $M \leq N$ copies of the same state in the past. In this case, the channel that we want to simulate is the partial trace over $(N-M)$ systems, symmetrized over all possible choices of the systems that are traced:

$\mathcal{C}_{N \rightarrow M}(\rho)=\operatorname{Tr}_{N} \operatorname{Tr}_{N-1} \ldots \operatorname{Tr}_{M+1}\left[\frac{1}{N !} \sum_{\pi \in S_{N}} U_{\pi}^{(N)} \rho U_{\pi}^{(N) \dagger}\right]$

Here the partial trace is over all input Hilbert spaces except the first $M, \pi$ is an element of the group $S_{N}$ of the permutations of $N$ objects and $U_{\pi}^{(N)}$ is the unitary operator that permutes the $N$ input Hilbert spaces according to the permutation $\pi$.
The goal will be to see how the probability of success, denoted by $p_{q, N \rightarrow M}^{+}$, varies with the number of input and output copies. In particular, we will show that the probability of success increases with the number of available input copies.

Note that in the classical world there cannot be any increase of the probability of success with the number of copies of an pure input state: Since we can perfectly clone the classical pure states corresponding to an orthonormal basis, we must have

$$
p_{c l, N \rightarrow M}^{+}=p_{c l, 1 \rightarrow 1}^{+}=\frac{1}{d} \quad \forall M, N .
$$

The classical value $p_{c l}=\frac{1}{d}$ is clearly an upper bound for the probability of success in the quantum case: Indeed, if we have a probabilistic teleportation protocol that succeeds with probability $p_{q, N \rightarrow M}^{+}$in transforming $N$ copies of a pure quantum state into $M$ copies of the same quantum state, then the protocol will work in particular on the states of an orthonormal basis, thus simulating the classical trace channel from $N$ to $M$ copies. Hence, we must have

$$
p_{q, N \rightarrow M}^{+} \leq \frac{1}{d} \quad \forall N, M .
$$

This bound is very intuitive: If there existed a quantum protocol that succeeded with probability $p_{q, N \rightarrow M}^{+}>\frac{1}{d}$, then two parties, Alice and Bob, could use the protocol to reliably win a lottery where the goal is to guess today a winning number $n$ between 1 and $d$ that will be extracted tomorrow. To win the lottery, Alice and Bob can agree to use an orthonormal basis $\{|n\rangle\}_{n=1}^{d}$ such that the basis vector $|n\rangle$ corresponds to the number $n$. Tomorrow, when the winning number is announced, Bob inputs into the teleportation machine $N$ identical systems, each of which is prepared in the state $|n\rangle$ corresponding to the winning number. Today Alice measures one of the $M$ copies in the agreed basis and with probability $p_{q, N \rightarrow M}^{+}$she obtains the winning number, just in time to submit her lottery ticket. If $p_{q, N \rightarrow M}^{+}$were larger than the classical value $p_{c l}=1 / d$, then Alice would win the lottery with probability larger than the probability $p_{\text {ran }}=\frac{1}{d}$ to win the lottery by a random guess.

In the following we will show that, unlike in the classical case, in the quantum case the probability of successful teleportation increases with the number of input copies. First, we will consider the simplest case $N=2, M=1$, where the calculation of the maximum probability of success can be done explicitly even without constraining the input copies to be in a pure state. In this case, we find out that the probability increases from the value $p_{1 \rightarrow 1}=\frac{1}{d^{2}}$ in the $N=1$ case to the value $p_{2 \rightarrow 1}=\frac{2}{d(d+1)}$. This result suggests that the quantum improvement is a general feature that does not depend on the assumption of pure input states. We will then move to the case of general $N$, keeping the number of output copies fixed to $M=1$. In this case, we will that the success probability approaches 
the classical value $p_{c l}=\frac{1}{d}$ as $N \rightarrow \infty$. An explanation of this asymptotic behaviour will be provided in Sec. VI.

Finally, we will extend the analysis to general $M$, also including the case $M>N$, where optimal clones of the input state are teleported to the past. Surprisingly, in the case of cloning channels we will show that for fixed $N$ the probability of success is an increasing function of the number of output copies $M$. Also this fact will be explained in Sec. VI.

\section{A. From two copies of a mixed state in the future to one copy in the past}

For two copies of input and one copy of output, the input Hilbert space is $\mathcal{H}_{i n}=\mathcal{H}^{\otimes 2}$ and the output Hilbert space is $\mathcal{H}_{\text {out }}=\mathcal{H} \simeq \mathbb{C}^{d}$. In this case, the quantum channel to be simulated is

$$
\mathcal{C}_{q, 2 \rightarrow 1}=\frac{1}{2}\left(\mathcal{I}_{1} \operatorname{Tr}_{2}+\mathcal{I}_{2} \operatorname{Tr}_{1}\right),
$$

where the labels 1 and 2 label on the right hand side label the two input spaces. Using Eq. (8), we find that the Choi operator of this channel is given by

$$
C_{q, 2 \rightarrow 1}=\frac{1}{2}(|I\rangle\rangle\left\langle\left\langle\left. I\right|_{01} \otimes I_{2}+\mid I\right\rangle\right\rangle\left\langle\left\langle\left. I\right|_{02} \otimes I_{1}\right),\right.
$$

where we have used 0 to label the output Hilbert space. Plugging this into the causality bound of Eq. (11) we obtain

$$
p \frac{1}{2}(|I\rangle\rangle\left\langle\left\langle\left. I\right|_{01} \otimes I_{2}+\mid I\right\rangle\right\rangle\left\langle\left\langle\left. I\right|_{02} \otimes I_{1}\right) \leq \rho_{0} \otimes I_{12}\right.
$$

Like in subsection IIIB, we can notice a symmetry of the Choi operator:

$$
\left(U \otimes U^{*} \otimes U^{*}\right) C_{q, 2 \rightarrow 1}\left(U^{\dagger} \otimes U^{T} \otimes U^{T}\right)=C_{q, 2 \rightarrow 1},
$$

for every $U \in S U(d)$. Applying this transformation to both sides of the inequality (25) and integrating over the Haar measure we obtain

$$
p \frac{1}{2}(|I\rangle\rangle\left\langle\left\langle\left. I\right|_{01} \otimes I_{2}+\mid I\right\rangle\right\rangle\left\langle\left\langle\left. I\right|_{02} \otimes I_{1}\right) \leq \frac{1}{d} I_{0} \otimes I_{12} .\right.
$$

It now remains to find the eigenvalues of the Choi operator $C_{q, 2 \rightarrow 1}$. To do this, we define the following set of orthonormal vectors in $\mathcal{H}_{\text {out }} \otimes \mathcal{H}_{\text {in }}$ :

$$
\begin{aligned}
\left|n_{+}\right\rangle & \left.\left.=\frac{1}{\sqrt{2(d+1)}}(|I\rangle\rangle_{01}|n\rangle_{2}+|I\rangle\right\rangle_{02}|n\rangle_{1}\right) \\
\left|n_{-}\right\rangle & \left.\left.=\frac{1}{\sqrt{2(d-1)}}(|I\rangle\rangle_{01}|n\rangle_{2}-|I\rangle\right\rangle_{02}|n\rangle_{1}\right) .
\end{aligned}
$$

In terms of these vectors, the Choi operator can be rewritten as

$$
C_{q, 2 \rightarrow 1}=\frac{d+1}{2} \sum_{n}\left|n_{+}\right\rangle\left\langle n_{+}\left|+\frac{d-1}{2} \sum_{n}\right| n_{-}\right\rangle\left\langle n_{-}\right|
$$

Thus we see that the eigenvalues of this Choi operator are $\gamma_{\max }=\frac{d+1}{2}$ and $\gamma_{\min }=\frac{d-1}{2}$. To ensure that Eq. 25. holds, we must have $p \gamma_{\max } \leq \frac{1}{d}$. Thus, the maximum teleportation probability from 2 copies of input is

$$
p_{q, 2 \rightarrow 1}=\frac{2}{d(d+1)},
$$

which is greater than the maximum probability of teleportation from 1 to 1 copy, $p_{q, 1 \rightarrow 1}=\frac{1}{d^{2}}$. In other words, adding an extra copy of the input yields improvement in the teleportation probability.

It is worth stressing that, since we are simulating the symmetrized trace channel $\mathcal{C}_{q, 2 \rightarrow 1}$, any bipartite state $\sigma$ on $\mathcal{H}_{i n}$ with the property $\left(\operatorname{Tr}_{1}[\sigma]+\operatorname{Tr}_{2}[\sigma]\right) / 2=\rho$ will be transformed with probability $p_{q, 2 \rightarrow 1}=\frac{2}{d(d+1)}$ into $\rho$. This holds in particular when $\sigma=\rho \otimes \rho$, corresponding to two identical copies of the mixed state $\rho$.

\section{B. From $N$ copies of a pure state in the future to $M=1$ copy in the past}

We now calculate the maximum probability of teleportation from $N$ input copies to one output copy. In this case, we will only require the teleportation protocol to work perfectly when we are inputting $N$ copies of the same pure state. Since the density matrix of $N$ identical copies of a pure state has support in the symmetric subspace of $\mathcal{H}^{\otimes N}$, henceforth denoted as $\left(\mathcal{H}^{\otimes N}\right)_{+}$, we will focus on the restriction of the trace channel to the symmetric subspace, namely on the $\operatorname{channel} \mathcal{C}_{q, N \rightarrow 1}^{+}$given by

$$
\mathcal{C}_{q, N \rightarrow 1}^{+}(\rho):=\mathcal{C}_{q, N \rightarrow 1}\left(P_{+}^{(N)} \rho P_{+}^{(N)}\right)
$$

where $P_{+}^{(N)}$ is the projector on $\left(\mathcal{H}^{\otimes N}\right)_{+}$and $\mathcal{C}_{q, N \rightarrow 1}$ is the symmetrized partial trace channel defined in Eq. 22 . Note that the map $\mathcal{C}_{q, N \rightarrow 1}^{+}$is trace-preserving only for states with support in $\left(\mathcal{H}^{\otimes N}\right)_{+}$.

It is useful to observe that, due to the projection on the symmetric subspace, the sum over all permutations in Eq. 22 can be omitted: Indeed, we have

$$
\begin{aligned}
\mathcal{C}_{q, N \rightarrow 1}^{+} & (\rho)= \\
& =\operatorname{Tr}_{N} \ldots \operatorname{Tr}_{2}\left[\frac{1}{N !} \sum_{\pi \in S_{N}} U_{\pi}^{(N)} P_{+}^{(N)} \rho P_{+}^{(N)} U_{\pi}^{(N) \dagger}\right] \\
& =\operatorname{Tr}_{N} \ldots \operatorname{Tr}_{2}\left[P_{+}^{(N)} \rho P_{+}^{(N)}\right]
\end{aligned}
$$

having used the property $U_{\pi}^{(N)} P_{+}^{(N)}=P_{+}^{(N)} U_{\pi}^{(N) \dagger}=$ $P_{+}^{(N)}$ for every permutation $\pi \in S_{N}$.

By the definition of Choi operator in Eq. (8) we find that the Choi operator $C_{q, N \rightarrow 1}^{+}$is given by

$$
C_{q, N \rightarrow 1}^{+}=\left(I_{0} \otimes P_{+}^{(N)}\right)(|I\rangle\rangle\left\langle\left\langle\left. I\right|_{01} \otimes P_{+}^{(N-1)}\right)\left(I_{0} \otimes P_{+}^{(N)}\right),\right.
$$




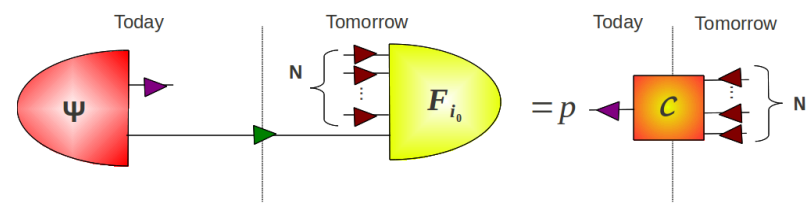

FIG. 4. (Color online) Probabilistic teleportation from $N$ copies to $M=1$ copy of a pure state. Here, $\mathcal{C}$ represents the symmetrized partial trace over all but one input copy, restricted to the symmetric subspace.

where the output Hilbert space is labelled by 0 , the input Hilbert spaces are labelled with numbers from 1 to $N$, and the projector $P_{+}^{(N-1)}$ acts on the tensor product of all input Hilbert spaces except the first.

We want to find the maximum value $p_{q, N \rightarrow 1}$ compatible with the causality bound of Eq. (11), which now reads

$$
p C_{q, N \rightarrow 1}^{+} \leq \rho_{\text {out }} \otimes I_{\text {in }} .
$$

To this purpose, we notice that the Choi operator $C_{q, N \rightarrow 1}^{+}$has the symmetry

$$
\left(U \otimes U^{* \otimes N}\right) C_{q, N \rightarrow 1}^{+}\left(U^{\dagger} \otimes U^{T \otimes N}\right)=C_{q, N \rightarrow 1}^{+},
$$

for every $U \in S U(d)$. Following again the group theoretic argument of subsection IIIB we can rewrite Eq. (28) as

$$
p C_{q, N \rightarrow 1}^{+} \leq \frac{1}{d} I_{\text {out }} \otimes I_{\text {in }} .
$$

Hence, it only remains to find the eigenvalues of $C_{q, N \rightarrow 1}^{+}$.

Expanding the projector $P_{+}^{(N-1)}$ as

$$
P_{+}^{(N-1)}=\sum_{n=1}^{d_{+}^{(N-1)}}\left|\varphi_{n}\right\rangle\left\langle\varphi_{n}\right|,
$$

where $\left\{\left|\varphi_{n}\right\rangle\right\}_{n=1}^{d_{+}^{(N-1)}}$ is an orthonormal basis for $\left(\mathcal{H}^{\otimes(N-1)}\right)_{+}$, we can express $C_{q, N \rightarrow 1}^{+}$as

$$
\begin{aligned}
& C_{q, N \rightarrow 1}^{+}= \\
& \quad=\left(I_{0} \otimes P_{+}^{(N)}\right)\left(\sum_{n=1}^{d_{+}^{(N-1)}}|I\rangle\right\rangle\left\langle\left\langle\left. I\right|_{01} \otimes \mid \varphi_{n}\right\rangle\left\langle\varphi_{n}\right|\right)\left(I_{0} \otimes P_{+}^{(N)}\right) \\
& \quad=\sum_{n=1}^{d_{+}^{(N-1)}}\left|\Phi_{n}\right\rangle\left\langle\Phi_{n}\right|
\end{aligned}
$$

having defined

$$
\left.\left|\Phi_{n}\right\rangle:=\left(I_{0} \otimes P_{+}^{(N)}\right)|I\rangle\right\rangle_{01}\left|\varphi_{n}\right\rangle .
$$

Eq. (30) is the desired diagonalization of the Choi operator $C_{q, N \rightarrow 1}^{+}$: Indeed, we have

$$
\begin{aligned}
\left\langle\Phi_{n} \mid \Phi_{m}\right\rangle & =\left\langle\left\langle\left. I\right|_{01}\left\langle\varphi_{n}\left|\left(I_{0} \otimes P_{+}^{(N)}\right)\right| I\right\rangle\right\rangle_{01} \mid \varphi_{m}\right\rangle \\
& =\left\langle\varphi_{n}\left|\operatorname{Tr}_{1}\left[P_{+}^{(N)}\right]\right| \varphi_{m}\right\rangle \\
& =\frac{d_{+}^{(N)}}{d_{+}^{(N-1)}}\left\langle\varphi_{n}\left|P_{+}^{(N-1)}\right| \varphi_{m}\right\rangle \\
& =\frac{d_{+}^{(N)}}{d_{+}^{(N-1)}} \delta_{n, m},
\end{aligned}
$$

that is, the vectors $\left\{\left|\Phi_{n}\right\rangle\right\}_{n=1}^{d_{+}^{(N-1)}}$ are mutually orthogonal and have the same norm. Therefore, $C_{q, N \rightarrow 1}^{+}$only has one non-zero eigenvalue, given by $\gamma_{N \rightarrow 1}=\frac{d_{+}^{(N)}}{d_{+}^{(N-1)}}$. Plugging this value into the causality bound of Eq. 29 we obtain $p \gamma_{N \rightarrow 1} \leq \frac{1}{d}$. The maximum probability of success is then given by

$$
\begin{aligned}
p_{q, N \rightarrow 1} & =\frac{d_{+}^{(N-1)}}{d d_{+}^{(N)}} \\
& =\frac{N}{d(d-1+N)} .
\end{aligned}
$$

Note that the probability of successful teleportation increases with the number $N$ of input copies, unlike in the classical case. For $N=1$ we retrieve the value $p_{q}=\frac{1}{d^{2}}$ of the standard teleportation protocol, while in the limit of $N$ going to infinity we observe that $p_{q, N \rightarrow 1}$ tends towards the classical limit $p_{c l}=\frac{1}{d}$. Such an asymptotic behaviour will be explained in section VI

\section{From $N$ copies of a pure state in the future to $M \leq N$ copies of the same state in the past}

Here we calculate the maximum probability of teleportation from $N$ input copies to $M \leq N$ output copies of a generic pure state. Again, since for every integer number $k$ the density matrix of $k$ identical copies of a pure state has support in the symmetric subspace $\left(\mathcal{H}^{\otimes k}\right)_{+}$, we will restrict the input of the partial trace channel $\mathcal{C}_{q, N \rightarrow M}$ to be in $\mathcal{H}_{\text {in }}:=\left(\mathcal{H}^{\otimes N}\right)_{+}$and the output to be in $\mathcal{H}_{\text {out }}:=\left(\mathcal{H}^{\otimes M}\right)_{+}$. In other words, we will focus on the probabilistic simulation of the channel $\mathcal{C}_{q, N \rightarrow M}^{+}$given by

$$
\mathcal{C}_{q, N \rightarrow M}^{+}(\rho):=P_{+}^{(M)}\left[\mathcal{C}_{q, N \rightarrow M}\left(P_{+}^{(N)} \rho P_{+}^{(N)}\right)\right] P_{+}^{(M)},
$$

where $\mathcal{C}_{q, N \rightarrow M}$ is the symmetrized partial trace channel defined in Eq. (22).

Again, thanks to the projection on the symmetric subspace we can write

$\mathcal{C}_{q, N \rightarrow M}^{+}(\rho):=P_{+}^{(M)}\left(\operatorname{Tr}_{N} \ldots \operatorname{Tr}_{M+1}\left[P_{+}^{(N)} \rho P_{+}^{(N)}\right]\right) P_{+}^{(M)}$. 


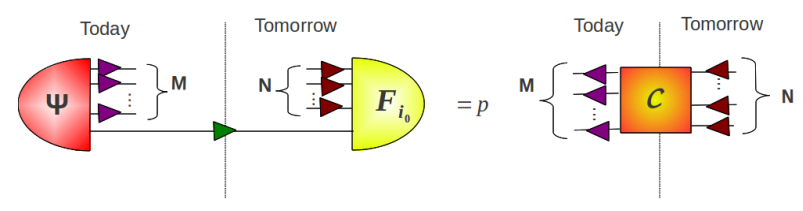

FIG. 5. (Color online) Probabilistic teleportation from with $N$ input copies in the future to $M \leq N$ output copies in the past. Here, $\mathcal{C}$ represents the symmetrized partial trace over all but $M$ of the $N$ input systems, where the input and output are constrained to be in the symmetric subspace.

By the definition of Choi operator in Eq. (8), we then obtain

$$
\begin{aligned}
C_{q, N \rightarrow M}^{+} & =\left(P_{+}^{(M)} \otimes P_{+}^{(N)}\right) K\left(P_{+}^{(M)} \otimes P_{+}^{(N)}\right) \\
K & =\left(\prod_{i=1}^{M}|I\rangle\right\rangle\left\langle\left\langle\left. I\right|_{i i} \otimes P_{+}^{(N-M)}\right)\right.
\end{aligned}
$$

where the projector $P_{+}^{(N-M)}$ acts on the tensor product of the last $N-M$ input Hilbert spaces.

Our goal is to find the maximum probability of success compatible with the causality bound

$$
p C_{q, N \rightarrow M}^{+} \leq \rho_{\text {out }} \otimes I_{\text {in }},
$$

where $\rho_{\text {out }}$ is a suitable state on $\mathcal{H}_{\text {out }}=\left(\mathcal{H}^{\otimes M}\right)_{+}$and $I_{\text {in }}=P_{+}^{(N)}$ is the identity on $\mathcal{H}_{\text {in }}=\left(\mathcal{H}^{\otimes N}\right)_{+}$. Now, it is easy to see that the Choi operator $C_{q, N-M}^{+}$has the symmetry

$$
\left(U^{\otimes M} \otimes U^{* \otimes N}\right) C_{q, N-M}^{+}\left(U^{\dagger \otimes M} \otimes U^{T \otimes N}\right)=C_{q, N-M}^{+},
$$

for every $U \in S U(d)$. Due to this symmetry, the state $\rho_{\text {out }}$ in the causality bound can be chosen without loss of generality to be the invariant state $\rho_{\text {out }}=\frac{I_{\text {out }}}{d_{\text {out }}}=\frac{P_{+}^{(M)}}{d_{+}^{(M)}}$, so that the bound becomes

$$
p C_{q, N \rightarrow M}^{+} \leq \frac{P_{+}^{(M)} \otimes P_{+}^{(N)}}{d_{+}^{(M)}} .
$$

To find the maximum probability it is enough to find the maximum eigenvalue of $C_{q, N \rightarrow M}$. Expanding the projector $P_{+}^{(N-M)}$ as

$$
P_{+}^{(N-M)}=\sum_{n=1}^{d_{+}^{(N-M)}}\left|\varphi_{n}\right\rangle\left\langle\varphi_{n}\right|
$$

for some orthonormal basis $\left\{\left|\varphi_{n}\right\rangle\right\}_{n=1}^{d_{+}^{(N-M)}}$ for $\left(\mathcal{H}^{\otimes(N-M)}\right)_{+}$, we can rewrite Eq. 32 as

$$
\begin{aligned}
C_{q, N \rightarrow M}^{+} & =\sum_{n=1}^{d_{+}^{(N-M)}} P_{+}^{M \otimes N}\left(\bigotimes_{i=1}^{M}|I\rangle\right\rangle\left\langle\left\langle\left. I\right|_{i i} \otimes \mid \varphi_{n}\right\rangle\left\langle\varphi_{n}\right|\right) P_{+}^{M \otimes N} \\
& =\sum_{n=1}^{d_{+}^{(N-M)}}\left|\Phi_{n}\right\rangle\left\langle\Phi_{n}\right|
\end{aligned}
$$

having defined $P_{+}^{M \otimes N}:=P_{+}^{(M)} \otimes P_{+}^{(N)}$ and

$$
\left.\left.\left.\left|\Phi_{n}\right\rangle:=P_{+}^{M \otimes N}\left(\left|I^{\otimes M}\right\rangle\right\rangle \otimes\left|\varphi_{n}\right\rangle\right) \quad\left|I^{\otimes M}\right\rangle\right\rangle:=\bigotimes_{i=1}^{M}|I\rangle\right\rangle_{i i} .
$$

Eq. (34) is the desired diagonalization of the Choi operator $C_{q, N \rightarrow M}^{+}$: Indeed, we have

$$
\begin{aligned}
\left\langle\Phi_{m} \mid \Phi_{n}\right\rangle & =\left\langle\left\langle I^{\otimes M} \mid\left\langle\varphi_{m}\left|\left(P_{+}^{(M)} \otimes P_{+}^{(N)}\right)\right| I^{\otimes M}\right\rangle\right\rangle \mid \varphi_{n}\right\rangle \\
& =\left\langle\varphi_{m}\left|\operatorname{Tr}_{1, \ldots, M}\left[P_{+}^{(N)}\right]\right| \varphi_{n}\right\rangle \\
& =\frac{d_{+}^{(N)}}{d_{+}^{(N-M)}}\left\langle\varphi_{m}\left|P_{+}^{(N-M)}\right| \varphi_{n}\right\rangle \\
& =\frac{d_{+}^{(N)}}{d_{+}^{(N-1)}} \delta_{m n}
\end{aligned}
$$

that is, the vectors $\left\{\left|\Phi_{n}\right\rangle\right\}$ are mutually orthogonal and have the same norm. Therefore, $C_{q, N \rightarrow M}^{+}$only has one non-zero eigenvalue, given by $\gamma_{N \rightarrow M}=\frac{d_{+}^{(N)}}{d_{+}^{(N-M)}}$.

The causality bound of Eq. (33) is equivalent to $p \gamma_{N \rightarrow M} \leq \frac{1}{d_{+}^{(M)}}$, which implies that the maximum probability is given by

$$
p_{q, N \rightarrow M}=\frac{d^{(N-M)}}{d_{+}^{(N)} d_{+}^{(M)}} .
$$

Note that $p_{q, N \rightarrow M}$ converges to the value

$$
p_{q, \infty \rightarrow M}=\frac{1}{d_{+}^{(M)}}
$$

in the limit of $N$ going to infinity. Such an asymptotic behaviour will be explained in Section VI

\section{From $N$ copies of a pure state in the future to $M>N$ optimal clones in the past}

We now generalize the results of the previous subsection to the case $M>N$. Of course, in this case we cannot expect to obtain $M$ perfect output copies of the input state, because that would violate the no-cloning theorem [18, 19]. What we can do is to consider the best possible approximation of the ideal cloning process. Such an optimal approximation is given by the optimal $u n i$ versal cloning channel [15], here denoted by $\mathcal{C}_{q, N \rightarrow M}^{+}$for consistency of notation. The cloning channel $\mathcal{C}_{q, N \rightarrow M}^{+}$is given by

$\mathcal{C}_{q, N \rightarrow M}^{+}(\rho)=\frac{d_{+}^{(N)}}{d_{+}^{(M)}} P_{+}^{(M)}\left(P_{+}^{(N)} \rho P_{+}^{(N)} \otimes I^{\otimes(M-N)}\right) P_{+}^{(M)}$

Note that the linear map $\mathcal{C}_{q, N \rightarrow M}^{+}$is trace-preserving only for density matrices $\rho$ with support within the symmetric 
subspace $\left(\mathcal{H}^{\otimes N}\right)_{+}$. Indeed, $\mathcal{C}_{q, N \rightarrow M}^{+}$should be regarded as a quantum channel sending states on $\mathcal{H}_{i n}:=\left(\mathcal{H}^{\otimes N}\right)_{+}$ to states on $\mathcal{H}_{\text {out }}:=\left(\mathcal{H}^{\otimes M}\right)_{+}$.

From Eqs. (31) and (36), we can note the existence of an important duality between universal cloning and partial trace: for two arbitrary operators $\nu$ on $\left(\mathcal{H}^{\otimes N}\right)_{+}$ and $\mu$ on $\left(\mathcal{H}^{\otimes M}\right)_{+}$one has

$$
\operatorname{Tr}\left[\mu \mathcal{C}_{q, N \rightarrow M}^{+}(\nu)\right]=\frac{d_{+}^{(M)}}{d_{+}^{(N)}} \operatorname{Tr}\left[\mathcal{C}_{q, M \rightarrow N}^{+}(\mu) \nu\right] .
$$

In other words, the map representing the universal cloning from $N$ to $M$ copies in the Heisenberg picture is the map representing the partial trace from $M$ to $N$ copies in the Schrödinger picture, up to a positive scaling factor. We will refer to this property as the time-reversal duality between partial trace and universal cloning.

In terms of Choi operators, the duality can be written as

$$
C_{q, N \rightarrow M}^{+}=\frac{d_{+}^{(N)}}{d_{+}^{(M)}} S_{N, M} C_{q, M \rightarrow N}^{+} S_{N, M}
$$

where $S_{N, M}$ is the operator that exchanges the $N$ output Hilbert spaces with the $M$ input Hilbert spaces for the channel $\mathcal{C}_{q, M \rightarrow N}^{+}$.

Eq. (38) allows us to easily find the probability of success for the cloning channel $\mathcal{C}_{q, N \rightarrow M}^{+}$. First of all, Eq. 38 implies that $C_{q, N \rightarrow M}^{+}$has the symmetry $\left(U^{\otimes M} \otimes U^{* \otimes N}\right) C_{q, N \rightarrow M}^{+}\left(U^{\otimes M} \otimes U^{* \otimes N}\right)=C_{q, N \rightarrow M}^{+}$for every $U \in S U(d)$. Using this symmetry the causality bound becomes

$$
p C_{q, N \rightarrow M}^{+} \leq \frac{P_{+}^{(M)} \otimes P_{+}^{(N)}}{d_{+}^{(M)}}
$$

Now, Eq. 38 implies that the above equation is equivalent to

$$
p C_{q, M \rightarrow N}^{+} \leq \frac{P_{+}^{(N)} \otimes P_{+}^{(M)}}{d_{+}^{(N)}},
$$

which is nothing but the causality bound for the trace channel $\mathcal{C}_{q, M \rightarrow N}^{+}$[cf. Eq. 33]]. Therefore, we have that the causality bound for the cloning channel $\mathcal{C}_{q, N \rightarrow M}^{+}$is satisfied with probability $p$ if and only if the causality bound for the trace channel $\mathcal{C}_{q, M \rightarrow N}^{+}$is satisfied with probability $p$. Hence, we have

$$
p_{q, N \rightarrow M}^{+}=p_{q, M \rightarrow N}^{+}=\frac{d_{+}^{(M-N)}}{d_{+}^{(M)} d_{+}^{(N)}} .
$$

Quite surprisingly, the above value increases with the number $M$ of output copies, reaching the asymptotic value

$$
p_{q, M \rightarrow \infty}=\frac{1}{d_{+}^{(N)}}
$$

in the limit $M \rightarrow \infty$. This asymptotic behaviour will be explained in Section VI.

\section{DEPENDENCE OF THE PROBABILITY OF SUCCESS ON THE AMOUNT AND TYPE OF INFORMATION TRANSMITTED BY THE CHANNEL}

The results obtained so far indicate a dependence of the maximum probability of success on the amount and type (quantum or classical) of information that the channel can transmit: First, we noted that the only channels that can be simulated with unit probability are the erasure channels, that produce a fixed output state independently of the input, - that is, they do not transmit any information. Then, we saw that measure-andprepare channels, that only transmit classical information, can be simulated with probability $p_{m \& p} \geq 1 / d_{\text {out }}$, $d_{\text {out }}$ being the dimension of the output Hilbert space. Finally, channels that transmit both quantum and classical information can be simulated with probability $p \geq$ $\max \left\{1 / d_{\text {in }}^{2}, 1 / d_{\text {out }}^{2}\right\}$, as in Eq. 16

In the following we will make a quantitative connection between the amount of information transmitted by a channel and the probability of simulating it with a generalized teleportation protocol: we will show that the probability of success is upper bounded by the inverse of a measure of information defined as the maximum payoff in a communication game. We will then use this result to explain the asymptotic behaviour of the probability of success for the simulation of a partial trace channel with a large number of input systems and of a universal cloning channel with a large number of output systems.

\section{A. Statistical information bounds}

In this subsection we relate probability of success in the simulation of a channel to the past with the amount of information transmitted by the channel. In this context, a convenient measure of information is the expected payoff obtained by two parties in a communication game.

Suppose that the channel $\mathcal{C}$ is used to transfer states from Alice's side to states on Bob's side. Alice can encode a set of messages $X$ in a set of states $\left\{\rho_{i}\right\}_{i \in X}$ on $\mathcal{H}_{\text {in }}$ and communicate a message to Bob by sending the corresponding state through the channel $\mathcal{C}$. On his side, Bob can try to decode Alice's message from the output state $\mathcal{C}\left(\rho_{i}\right)$. Generally, the decoding will be described by a POVM $\left\{P_{j}\right\}_{j \in Y}$ on the output Hilbert space $\mathcal{H}_{\text {out }}$, with the outcome $y$ corresponding to the decoded message. Usually, but not necessarily, the set of decoded messages $X$ coincides with the set of encoded messages $Y$. To assess the success of the communication, we can introduce a payoff function $f: X \times Y \rightarrow \mathbb{R}$, that quantifies the gain (or the loss) associated to decoding $j$ while the encoded message was $i$. For given $i$, the expected 
payoff will be

$$
\mathbb{E}_{i}(f):=\sum_{j \in Y} f(i, j) \operatorname{Tr}\left[\mathcal{C}\left(\rho_{i}\right) P_{j}\right] .
$$

Now, suppose that Alice is in the future and Bob is in the past, and that they are using a probabilistic simulation of channel $\mathcal{C}$. The causality bound of Eq. (7) imposes $p \mathcal{C}\left(\rho_{i}\right) \leq \rho_{0}$, and, therefore

$$
p \mathbb{E}_{i}(f) \leq \mathbb{E}_{i, \rho_{0}}(f) \quad \forall i \in X,
$$

having defined $\mathbb{E}_{i, \rho_{0}}:=\sum_{j \in Y} f(i, j) \operatorname{Tr}\left[\rho_{0} P_{j}\right]$. Introducing a prior probability distribution $\left\{\pi_{i}\right\}_{i \in X}$ on Alice's messages we can then consider the average expected payoff

$$
\mathbb{E}_{\text {ave }}(f):=\sum_{i \in X} \pi_{i} \mathbb{E}_{i}(f)
$$

Hence, the bound of Eq. 39 becomes

$$
\begin{aligned}
p \mathbb{E}_{\text {ave }}(f) & \leq \sum_{i \in X} \pi_{i} \mathbb{E}_{i, \rho_{0}}(f) \\
& \leq \max _{j \in Y}\left(\sum_{i \in X} \pi_{i} f(i, j)\right):=f_{\pi} .
\end{aligned}
$$

Note that the value $f_{\pi}$ depends only on the payoff function $f$ and on the prior probabilities $\left\{\pi_{i}\right\}_{i \in X}$.

We have now arrived at the bound

$$
p_{\mathcal{C}} \leq \frac{f_{\pi}}{\mathbb{E}_{\text {ave }}(f)}
$$

which holds for every given payoff function $f$, prior probabilites $\left\{\pi_{i}\right\}$, encoding $\left\{\rho_{i}\right\}_{i \in X}$, and decoding $\left\{P_{j}\right\}_{j \in Y}$. Maximizing the average payoff over all possible choices of encoding $\left\{\rho_{i}\right\}_{i \in X}$ and decoding $\left\{P_{j}\right\}_{j \in Y}$ we then get the maximum value

$$
\mathbb{E}_{\max }(f):=\max _{\left\{P_{j}\right\}_{j \in Y}} \max _{\left\{\rho_{i}\right\}_{i \in X}} \mathbb{E}_{\text {ave }}(f),
$$

which quantifies the maximum payoff that can be achieved when using the channel $\mathcal{C}$ in a communication game with given payoff function $f$ and prior probabilities $\left\{\pi_{i}\right\}_{i \in X}$.

We then conclude that the maximum probability to simulate the channel $\mathcal{C}$, denoted by $p_{\mathcal{C}}$, has to satisfy the bound

$$
p_{\mathcal{C}} \leq \frac{f_{\pi}}{\mathbb{E}_{\max }(f)}
$$

for every payoff function $f$ and for every prior $\left\{\pi_{i}\right\}_{i \in X}$. The above bound states that the probability of success cannot be greater than the inverse of the maximum amount of statistical information (average payoff) transmitted by the channel. For this reason, we refer to this bound as the statistical information bound.
Notice that the derivation of the information bound in Eq. (40) can be extended to the case where Alice and Bob are allowed to communicate through an ideal quantum channel on a reference system with Hilbert space $\mathcal{H}_{R}$, in addition to the channel $\mathcal{C}$. In this case, Alice can encode her message $i \in X$ in a bipartite state $\rho_{i}$ on $\mathcal{H}_{\text {in }} \otimes \mathcal{H}_{R}$. Later, she will send the input system through the channel $\mathcal{C}$ and the reference through the ideal channel.

Bob's task will then be to decode the message from the output state $\left(\mathcal{C} \otimes \mathcal{I}_{R}\right)\left(\rho_{i}\right)$, through a bipartite POVM $\left\{P_{j}\right\}_{j \in Y}$ on $\mathcal{H}_{\text {out }} \otimes \mathcal{H}_{R}$. Following the same steps as above, in this case we obtain the entanglement-assisted statistical information bound

$$
p_{\mathcal{C}} \leq \frac{\mathbb{E}_{\text {ave }}^{(R)}(f)}{\mathbb{E}_{\text {ave }}^{(S R)}(f)},
$$

having defined

$$
\begin{aligned}
& \mathbb{E}_{\text {ave }}^{(S R)}(f):=\sum_{i \in X} \pi_{i}\left\{\sum_{j \in Y} f(i, j) \operatorname{Tr}\left[\left(\mathcal{C} \otimes \mathcal{I}_{R}\right)\left(\rho_{i}\right) P_{j}\right]\right\} \\
& \left.\mathbb{E}_{\text {ave }}^{(R)}(f):=\sum_{i \in X} \pi_{i}\left\{\sum_{j \in Y} f(i, j) \operatorname{Tr}\left[\rho_{0} \otimes \operatorname{Tr}_{i n}\left[\rho_{i}\right]\right) P_{j}\right]\right\} .
\end{aligned}
$$

Note, however, that both statistical information bounds are just upper bounds: In general there is no guarantee about their achievability.

\section{B. Asymptotic behaviour of the optimal probabilistic teleportation from $N$ to $M \leq N$ copies}

The aim of the following discussion is to provide an explanation for the asymptotic behaviour of the probability of success in the simulation of the partial trace channel $\mathcal{C}_{q, N \rightarrow M}^{+}$in the limit $N \rightarrow \infty$. First, we apply the statistical information bound of Eq. (40) to show that the probability of success satisfies the bound

$$
p_{q, N \rightarrow M}^{+} \leq \frac{1}{d_{+}^{(M)}} \quad \forall N \geq M .
$$

Consider a communication scenario where Alice encodes a message in state from the set $\left\{|\varphi\rangle^{\otimes N}\right\}_{|\varphi\rangle \in \mathcal{H}}$ and sends it to Bob through the partial trace channel $\mathcal{C}_{q, N \rightarrow M}$. Suppose that Bob's decoding is given by the POVM

$$
P_{\psi}^{(M)} d \psi=d_{+}^{(M)}(|\psi\rangle\langle\psi|)^{\otimes M} d \psi,
$$

where $d \psi$ is the normalized invariant measure on the pure states of $\mathcal{H}$. As a payoff function, let us assume the Dirac delta $f(\varphi, \psi)=\delta(\varphi-\psi)$, so that the expected payoff for input $|\varphi\rangle$ is just the probability (density) to make the correct guess

$$
\begin{aligned}
\mathbb{E}_{\varphi}(f) & =\int d \psi \delta(\varphi-\psi) \operatorname{Tr}\left\{\mathcal{C}_{q, N \rightarrow M}^{+}\left[(|\varphi\rangle\langle\varphi|)^{\otimes N}\right] P_{\psi}^{(M)}\right\} \\
& =\operatorname{Tr}\left[(|\varphi\rangle\langle\varphi|)^{\otimes M} P_{\varphi}^{(M)}\right] \\
& =d_{+}^{(M)} \quad \forall|\varphi\rangle \in \mathcal{H} .
\end{aligned}
$$


Since the expected payoff is the same for every state, we have

$$
\mathbb{E}_{\text {ave }}(f)=d_{+}^{(M)}
$$

for every prior distribution. On the other hand, choosing the uniform distribution $\pi(d \varphi)=d \varphi$ for the input states, we have

$$
\begin{aligned}
f_{\pi} & =\max _{\psi}\left[\int d \varphi \delta(\varphi-\psi)\right] \\
& =1 .
\end{aligned}
$$

The statistical information bound of Eq. 40 then gives $p_{q, N \rightarrow M}^{+} \leq \frac{1}{d_{+}^{(M)}}$.

In subsection $\mathrm{VC}$ we observed that the probability of success $p_{q, N \rightarrow M}^{+}$asymptotically approaches the value $p_{q, \infty \rightarrow M}^{+}=1 / d_{+}^{(M)}$ in the limit $N \rightarrow \infty$. An explanation of this behaviour is the following: From Ref. [21, we know that in the limit $N \rightarrow \infty$ the partial trace channel $\mathcal{C}_{q, N \rightarrow M}^{+}$converges to the estimation channel $\mathcal{C}_{\text {est }, N \rightarrow M}^{+}$ defined by

$$
\mathcal{C}_{\text {est }, N \rightarrow M}^{+}(\rho):=\int d \psi(|\psi\rangle\langle\psi|)^{\otimes M} \operatorname{Tr}\left[\rho P_{\psi}^{(N)}\right] .
$$

with $P_{\psi}^{(N)}:=d_{+}^{(N)}(|\psi\rangle\langle\psi|)^{\otimes N}$. Hence, the probability of success $p_{q, N \rightarrow M}^{+}$must converge to $p_{e s t, N \rightarrow M}^{+}$, the probability of success for the estimation channel $\mathcal{C}_{\text {est }, N \rightarrow M}^{+}$. On the other, hand, since $\mathcal{C}_{\text {est }, N \rightarrow M}^{+}$is a measure-andprepare channel, by the general bound of Eq. 18 we must have $p_{e s t, N \rightarrow M}^{+} \geq 1 / d_{+}^{(M)}$. In conclusion, we obtained

$$
\frac{1}{d_{+}^{(M)}} \geq \lim _{N \rightarrow \infty} p_{q, N \rightarrow M}^{+}=\lim _{N \rightarrow \infty} p_{e s t, N \rightarrow M}^{+} \geq \frac{1}{d_{+}^{(M)}},
$$

that is, $\lim _{N \rightarrow \infty} p_{q, N \rightarrow M}^{+}=\frac{1}{d_{+}^{(M)}}$. In other words, the asymptotic behaviour of $p_{q, N \rightarrow M}^{+}$is dictated by the statistical information bound and by the convergence of the trace channel $\mathcal{C}_{q, N \rightarrow M}^{+}$to a measure-and-prepare channel.

An illustration of the situation for the case of $M=$ 1 is given in Fig. 6. It is worth stressing, once more, that no improvement with the number of input copies would be possible for a classical partial trace channel: Since in that case the probability is already $p_{c l}=\frac{1}{d}$, any improvement would violate the causality bound for ideal classical channels. Hence, the increase of the probability of teleportation with the number $N$ of input copies is intrinsically a quantum phenomenon.

\section{Asymptotic behaviour of the optimal} probabilistic teleportation from $N$ to $M \geq N$ copies

Here we consider the universal cloning channel $\mathcal{C}_{q, N \rightarrow M}^{+}$. As we did for the trace channels, we will now

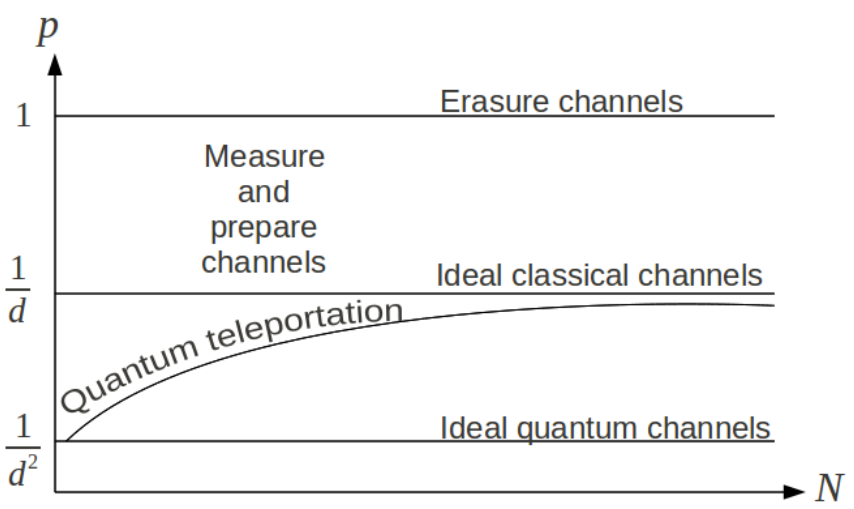

FIG. 6. A graph of the maximum probability $p$ to simulate the partial trace channel from $N$ copies to $M=1$ copy of a pure input state. The maximum probabilities for erasure channels, measure-and-prepare channels, and ideal classical and quantum channels are also located in the plot.

provide an explanation for the asymptotic behaviour of the probability of success in the limit $M \rightarrow \infty$.

A direct explanation comes from the time-reversal duality between cloning and partial trace, which implies the relation $p_{q, N \rightarrow M}^{+}=p_{q, M \rightarrow N}^{+}$and thus allows one to reduce the asymptotic behaviour of the probability of success of a cloning channel with many output copies to the asymptotic behaviour of the probability of success for a trace channel with many input copies.

A less direct, but perhaps more insightful explanation can be obtained by following the same steps used in the previous subsection. Let us start by showing that the statistical information bound of Eq. (40) implies that the probability of success satisfies the bound

$$
p_{q, N \rightarrow M}^{+} \leq \frac{1}{d_{+}^{(N)}} \quad \forall M \geq N .
$$

Consider a communication scenario where Alice encodes a message in state from the set $\left\{|\varphi\rangle^{\otimes N}\right\}_{|\varphi\rangle \in \mathcal{H}}$ and sends it to Bob through the cloning channel $\mathcal{C}_{q, N \rightarrow M}$. Suppose that Bob's decoding is given by the POVM $P_{\psi}^{(M)} d \psi=$ $d_{+}^{(M)}(|\psi\rangle\langle\psi|)^{\otimes M} d \psi$, where $d \psi$ is the normalized invariant measure on the pure states of $\mathcal{H}$. As a payoff function we assume again the Dirac delta $f(\varphi, \psi)=\delta(\varphi-\psi)$, so that the expected payoff for input $|\varphi\rangle$ is the probability (density) of the correct guess:

$$
\begin{aligned}
\mathbb{E}_{\varphi}(f) & =\int d \psi \delta(\varphi-\psi) \operatorname{Tr}\left\{\mathcal{C}_{q, N \rightarrow M}^{+}\left[(|\varphi\rangle\langle\varphi|)^{\otimes N}\right] P_{\psi}^{(M)}\right\} \\
& =\operatorname{Tr}\left\{\mathcal{C}_{q, N \rightarrow M}^{+}\left[(|\varphi\rangle\langle\varphi|)^{\otimes N}\right] P_{\varphi}^{(M)}\right\} \\
& =\frac{d_{+}^{(N)}}{d_{+}^{(M)}} \operatorname{Tr}\left[(|\varphi\rangle\langle\varphi|)^{\otimes N} \mathcal{C}_{q, M \rightarrow N}^{+}\left(P_{\varphi}^{(M)}\right)\right] \\
& =\operatorname{Tr}\left[(|\varphi\rangle\langle\varphi|)^{\otimes N} P_{\varphi}^{(N)}\right] \\
& =d_{+}^{(N)} \quad \forall|\varphi\rangle \in \mathcal{H} .
\end{aligned}
$$


Since the expected payoff is the same for every state, for every prior distribution we have $\mathbb{E}_{\text {ave }}(f)=d_{+}^{(N)}$. On the other hand, we already know that $f_{\pi}=1$, so that the information bound of Eq. 40 gives $p_{q, N \rightarrow M} \leq \frac{1}{d_{+}^{(N)}}$.

On the other hand, from Ref. 21] we know that in the limit $M \rightarrow \infty$ the cloning channel $\mathcal{C}_{q, N \rightarrow M}^{+}$converges to the estimation channel $\mathcal{C}_{e s t, N \rightarrow M}^{+}$. Hence, the probability of success $p_{q, N \rightarrow M}^{+}$must converge to $p_{e s t, N \rightarrow M}^{+}$. Again, we obtain the relation

$$
\frac{1}{d_{+}^{(N)}} \geq \lim _{M \rightarrow \infty} p_{q, N \rightarrow M}^{+}=\lim _{M \rightarrow \infty} p_{e s t, N \rightarrow M}^{+} \geq \frac{1}{d_{+}^{(N)}},
$$

that is, $\lim _{M \rightarrow \infty} p_{q, N \rightarrow M}^{+}=\frac{1}{d_{+}^{(N)}}$. Also in this case, the asymptotic behaviour of the probability $p_{q, N \rightarrow M}^{+}$is dictated by the statistical information bound and by the convergence of the cloning channel $\mathcal{C}_{q, N \rightarrow M}^{+}$to a measureand-prepare channel.

\section{CONCLUSIONS}

This work showed how causality determines the maximum probability of simulating a given quantum channel from the future to the past. Since causality states that it is not possible to signal from the future to the past, the probability of success in the simulation should be small enough to prevent any signal from being sent. As a consequence, channels that are able to transmit more information will have a smaller probability of being simulated, while channels that transmit less information will have a larger probability. In particular, erasure channels, that transmit no information at all, can be simulated with unit probability, while measure-and-prepare channels, that transmit only classical information, can be simulated with probability at least equal to $p_{c l}=1 / d_{\text {out }}$ the inverse of the output Hilbert space dimension. The hardest channels to simulate are the ideal quantum channels, for which the probability is equal to $p_{q}=1 / d^{2}$, the inverse of the square of the Hilbert space dimension. A quantitative connection between the amount of information transmitted by a channel and the probability of successful simulation is given by the statistical information bounds, which state that the probability of success is upper bounded by the inverse of the statistical information transmitted by the channel.

In the paper we computed explicitly the maximum probability for the simulation of several channels, including partial trace channels from $N$ input systems to $M \leq N$ output systems, and universal cloning channels from $N$ input systems to $M \geq N$ output systems. We pointed out a time-reversal duality between trace and cloning and we exploited it to show that the value of the maximum probability of success is given by $p_{q, N \rightarrow M}^{+}=d_{+}^{(|N-M|} /\left(d_{+}^{(M)} d_{+}^{(N)}\right)$. Note that for fixed $M$, the probability of success is an increasing function of $N$, the number of input copies. Similarly, for fixed $N$, the probability of success is an increasing function of $M$, the number of output copies. These are genuinely quantum features, that are impossible in the classical world: Indeed, classically the probability of success for all trace channels and for all cloning channels is given by $p_{c l}=1 / d$, independently of $N$ and $M$.

In the case of a single output copy, $M=1$ the probability of success for a quantum partial trace channel tends to the classical value $p_{c l}=1 / d$ in the limit of $N$ going to infinity. This asymptotic behaviour can be explained from the fact that a trace channel with asymptotically large number of input copies converges to a measureand-prepare channel 21]. The same explanation can be provided for the asymptotic behaviour of the probability of success for cloning channels. Also in this case the asymptotic behaviour is dictated by the fact that a universal cloning channel with asymptotically large number of output copies converges to a measure-and-prepare channel [21.

Note that in this paper we focused on determining the maximum probability of success for many channels, but we did not show explicitly the generalized teleportation schemes that achieve the simulation of those channels. In particular, it is interesting to ask which kind of entangled states and which kind of measurements allow one to achieve teleportation from $N$ input copies to $M$ output copies. Moreover, it is worth asking whether these probabilistic teleportation protocols can be extended to deterministic protocols (now, from the past to the future) by reintroducing classical communication and correction operations, as in the case of tele-cloning 28. The fact that the probability of successful teleportation from $N$ copies to $M=1$ copy converges to $p_{c l}=1 / d$ suggests that our probabilistic protocol could be extended to a deterministic protocol that needs only $\log (d)$ bits of classical communication, instead of the $2 \log (d)$ bits of the original teleportation protocol. However, it is quite possible that in general there exists a trade-off between maximizing the probability of success for a particular outcome and being able to implement a correction for all outcomes. All these issues will be investigated in a forthcoming work 29 .

Another interesting direction of future research is to consider the simulation of multipartite channels under the constraint that different input and output systems can arrive at different instants of time, and to show how the probability of simulation depends on the possible causal arrangements of the input/output systems. For example, one could consider the case of a cloning channel from $N=1$ input copy to $M=2$ approximate copies, one copy being sent to the past and the other being sent to the future. Examples of this kind are expected to shed light on the interplay between causal structure and quantum information flow. 


\section{ACKNOWLEDGMENTS}

Research at Perimeter Institute for Theoretical Physics is supported in part by the Government of Canada through NSERC and by the Province of Ontario through MRI.
[1] G. Chiribella, G. M. D'Ariano, and P. Perinotti, "Probabilistic theories with purification", Phys. Rev. A 81, 062348 (2010)

[2] G. Chiribella, G. M. D'Ariano, and P. Perinotti, "Informational derivation of quantum theory", Phys. Rev. A 84, 012311 (2011).

[3] C.H. Bennett, G. Brassard, C. Crepeau, R. Jozsa, A. Peres, and W.K. Wooters, "Teleporting an unknown quantum state via dual classical and Einstein-PodolskiRosen channels", Phys. Rev. Lett. 70, 1895 (1993).

[4] C.H. Bennett, talk at QUPON, Wien, May 2005; http://www.research.ibm.com/people/b/bennetc/ tifr.res.in/ qpip/HTML/Courses/Bennett/TIFR5.pdf

[5] G. Svetlichny, "Effective Quantum Time Travel", arXiv:0902.4898v1.

[6] G. T. Horowitz and J. Maldacena. "The black hole final state", JHEP 02, 008 (2004).

[7] D. Gottesmann and J. Preskill, Comment on "The black hole final state", JHEP 03, 026 (2004).

[8] S. Abramsky and B. Coecke, "A categorical semantics for quantum protocols", Proc. of LiCS'04, IEEE Computer Science Press (2004).

[9] B. Coecke, "Quantum Information flow, concretely, and axiomatically", arXiv:quant-ph/0506132

[10] S. Lloyd, L. Maccone, R. Garcia-Patron, V. Giovannetti, Y. Shikano, S. Pirandola, L. A. Rozema, A. Darabi, Y. Soudagar, L. K. Shalm, A. M. Steinberg "Closed timelike curves via post-selection: theory and experimental demonstration", Phys. Rev .Lett. 106, 040403, (2011)

[11] S. Lloyd, L. Maccone, R. Garcia-Patron, V. Giovannetti, and Y. Shikano, "The quantum mechanics of time travel through post-selected teleportation", Phys. Rev. D 84, 025007 (2011).

[12] R. D. da Silva, E. F. Galvão, and E. Kashefi, "Closed timelike curves in measurement-based quantum computation", Phys. Rev. A 83, 012316 (2011).

[13] M. Hillery and V. Bužek, "Quantum copying: Fundamental inequalities", Phys. Rev. A 56, 1212 (1997).

[14] N. Gisin and S. Massar, "Optimal Quantum Cloning Machines", Phys. Rev. Lett. 79, 2153 (1997).

[15] R.F. Werner, "Optimal Cloning of Pure States", Phys. Rev. A 58, 1827 (1998)
[16] N. J. Cerf, A. Ipe, X. Rottenberg, "Universal cloning of continuous quantum variables", Phys. Rev. Lett. 85,1754 (2000).

[17] G. M. DAriano and C. Macchiavello, "Optimal phasecovariant cloning for qubits and qutrits", Phys. Rev. A 67, 042306 (2003).

[18] W. K. Wooters and W. H. Zurek, "A single quantum cannot be cloned", Nature 299, 802 (1982)

[19] D. Dieks, "Communication by EPR devices", Phys. Lett. A 92, $271(1982)$

[20] V. Bužek, M. Hillery, and R. F. Werner, "Optimal manipulations with qubits: Universal-NOT gate", Phys. Rev. A 60, R 2626 (1999).

[21] G. Chiribella, "On quantum estimation, quantum cloning, and finite quantum de Finetti theorems", Theory of Quantum Computation, Communication, and Cryptography, Lecture Notes in Computer Science, 6519/2011, 9 (2011)

[22] M. Choi, "Completely Positive Linear Maps on Complex matrices", Linear Algebra and Its Applications 10, 285-290, 1975

[23] G. Chiribella, G. Mauro D'Ariano, and P. Perinotti, "Quantum Circuits Architecture", Phys. Rev. Lett. 101, 060401 (2008).

[24] G. Chiribella, G. Mauro D'Ariano, and P. Perinotti, "Theoretical framework for quantum networks", Phys. Rev. A 80, 022339 (2009)

[25] K. Kraus, "General state changes in quantum theory", Annals of Physics 64, 311 (1971)

[26] G.M. D'Ariano, P. Lo Presti, and M.F. Sacchi, "Bell measurements and observables", Phys. Lett. A 272, 32 (2000)

[27] A. S. Holevo and R. F. Werner,Evaluating the Capacity of Bosonic Quantum Channels", Phys. Rev. A 63, 32312 (2001).

[28] M. Murao, D. Jonathan, M. B. Plenio, and V. Vedral, "Quantum telecloning and multiparticle entanglement", Phys. Rev. A 59, 156161 (1999).

[29] D. Genkina, G. Chiribella, and L. Hardy, in preparation. 